\title{
Too severe to be functional? An unusual case of schizophrenia with catatonic, cognitive and negative symptoms.
}

L.A. Fernandes1, F. Ferreira ${ }^{1,}$ G. Marinho ${ }^{2}$, M.J. Santos ${ }^{1}$, D. Almeida ${ }^{1}$, A. Valverde ${ }^{2}$, N. Borja-Santos ${ }^{1}$, T. Maia ${ }^{1}$ ${ }^{1}$ Serviço de Psiquiatria do Hospital Prof. Doutor Fernando Fonseca, E.P.E ${ }^{2}$ Serviço de Neurologia do Hospital Prof. Doutor Fernando Fonseca ${ }^{3}$ Departamento de Psiquiatria e Saúde Mental, Centro Hospitalar Universitário do Algarve 区luis.fernandes@hff.min-saude.pt

\section{Background}

-Objectives: to describe an atypical schizophrenia case.

-Material and methods: case report.
- Background and aims: Schizophrenia is a multi-dimensional disorder with numerous possible presentations, such as the one we describe herein.

\section{Clinical Case}

\section{Identification}

32 years-old male, inpatient in a Day Hospital. 12 years of formal education (frequency of architecture bachelor). Poor development of communication skills.

\section{Psychiatric history}

Age

\section{9}

29

32
Anxiety symptoms:

Insidious psychotic symptoms

Follow-up in community mental healith team

\section{Px: Olanzapine}

Partial clinical response, with significant negative symptoms.

Biographical rupture. The patient didn't return to previous functioning, dropping-out from university and having no known job.

- Catatonia + delusions + hallucinations.

\section{Admission to inpatient ward}

Px: Electroconvulsive therapy \& clozapine

- Improvement of catatonic and positive symptoms.

- Residual motor symptoms. Severe cognitive and negative symptoms. Obsessive-compulsive symptoms.

- Bizarre visual symptoms:

- Aura-like

- Visual-field splicing.

- No functional recovery.

\section{Observation}

Positive for: Psychomotor retardation, motor stereotypies, alogia and blunted affect. Negative for delusions, and hallucinations. No Kayser-Fleischer ring. Preserved insight.

Positive and Negative Syndrome Scale $=89$ (Negative scale $=42$; General psychopatology= 38 ).

Personal and Social Performance Scale $=25$.

Addenbrooke's Cognitive Examination-III $=88$, with low score in memory and verbal fluency.

\section{Differential}

Posterior cortical atrophy: due to memory impairment and atypical visual symptoms.

Wilson's disease: due to motor symptoms, cognitive frontal impairment and psychosis.

Schizophrenia: with co-morbid obsessive-compulsive symptoms.

\section{Complementary exams}

- Serum clozapine under therapeutic range

- Recurrent folic acid deficiency.

- Normal urinary and serum cooper. Normal thyroid

- Negative results for HIV and syphilis.

- Electroencephalogram is normal.

- Magnetic resonance imaging of the brain with subtle bilateral parietal atrophy.

- Liquor biomarkers:

- F-18 Fluorodeoxyglucose Positron Emission Tomography (PET-FDG) with generalized mild reduction of FDG uptake.

\section{Discussion and conclusions}

- A wide differential diagnosis was considered concerning the present case; however none of the complementary exams findings favored an "organic" disorder as the cause for the symptoms.
Subsequently schizophrenia is the most probable diagnosis, albeit the unusual presentation. 